\title{
Distribution of KRAS and BRAF Mutations in Metastatic Colorectal Cancers in Turkish Patients
}

\author{
Orhan Gorukmez ${ }^{1}$, Tahsin Yakut ${ }^{2 *}$, Ozlem Gorukmez², Sebnem Ozemri Sag ${ }^{2}$, \\ Mutlu Karkucak ${ }^{3}$, Ozkan Kanat ${ }^{4}$
}

\begin{abstract}
The results of this study demonstrate the potential prognostic and predictive values of KRAS and BRAF gene mutations in patients with colorectal cancer (CRC). It has been proven that KRAS and BRAF mutations are predictive biomarkers for resistance to anti-EGFR monoclonal antibody treatment in patients with metastatic CRC (mCRC). We demonstrated the distribution of KRAS (codons 12, 13 and 61) and BRAF (codon 600) gene mutations in $50 \mathrm{mCRCs}$ using direct sequencing and compared the results with clinicopathological data. KRAS and BRAF mutations were identified in $15(30 \%)$ and $1(2 \%)$ patients, respectively. We identified KRAS mutations in codon 12, 13 and 61 in $73.3 \%(11 / 15), 20 \%(3 / 15)$ and $6.67 \%(1 / 15)$ of the positive patients, respectively. The KRAS mutation frequency was significantly higher in tumors located in the ascending colon $(p=0.043)$. Thus, we found that approximately $1 / 3$ of the patients with mCRC had KRAS mutations and the only clinicopathological factor related to this mutation was tumor location. Future studies with larger patient groups should yield more accurate data regarding the molecular mechanism of CRC and the association between KRAS and BRAF mutations and clinicopathological features.
\end{abstract}

Keywords: Metastatic colorectal cancer - mutations - BRAF - KRAS - clinicopathological variables

Asian Pac J Cancer Prev, 17 (3), 1175-1179

\section{Introduction}

Colorectal cancer (CRC) is one of the most common malignancies seen worldwide. It remains an important cause of mortality and morbidity despite the considerable developments in its treatment, and it is the fourth most common cause of cancer-related death worldwide (Brenner et al., 2014). Our improved understanding of CRC biology has led to the development of targeted molecular agents (Friday and Adjei, 2008). However, the use of these agents has generated significant differences in clinical responses. These differences emphasize the need for reliable biomarkers to predict the molecular heterogeneity of colorectal tumors and the therapeutic efficacy (Jean and Shah, 2008).

The epidermal growth factor receptor (EGFR) pathway is frequently upregulated in CRCs (Venook, 2005). EGFR is a transmembrane tyrosine kinase receptor that controls various cellular responses, such as apoptosis, differentiation, cellular migration and adhesion (Roberts and Der, 2007). EGFR tyrosine kinase and the downstream pathways have crucial roles in CRC carcinogenesis and represent a target for biological therapy in this disease. Various pathways, such as RAS / RAF / MEK / ERK, PI3K/Akt, JAK / STAT and PLC $\gamma$, are stimulated by EGFR signals. Cetuximab or panitumumab are monoclonal antibodies used in clinical practice to target EGFR, and they are chemotherapeutics or adjuvant agents for the treatment of mCRC (Cunningham et al., 2004). These monoclonal antibodies block signaling downstream of EGFR.

One of the downstream pathways is the RAS-RAFMAPK axis, which has a role in cellular proliferation. Another downstream pathway is the PI3K-PTEN-AKT axis, which is a critical mediator for cellular motility, invasion and survival (Ciardiello and Tortora, 2008). Approximately $35 \%$ of CRC tissues have a mutation in KRAS codon 12 or 13 , which leads to activation in downstream pathways of EGFR (Di Nicolantonio et al., 2008; Marchoudi et al., 2013). Identifying the KRAS/ BRAF genotypes aids in choosing systemic chemotherapy in individuals with advanced stage or recurrent CRC and may identify patients with poor prognosis. The KRAS and BRAF genes are potential prognostic markers and are predictive biomarkers in patients with $\mathrm{mCRC}$ treated with anti-EGFR monoclonal antibodies, such as cetuximab and panitumumab. Various retrospective studies have shown that cetuximab is ineffective in patients with KRAS mutations. Thus, KRAS genotype is a helpful predictive biomarker for cetuximab or panitumumab treatments 
Orhan Gorukmez et al

of CRC (Yokota, 2012). Moreover, wild type BRAF is needed for a successful response to either cetuximab or panitumumab treatments in patients with mCRC (Di Nicolantonio et al., 2008). In this study, we demonstrated the distribution of KRAS and BRAF gene mutations in patients with mCRC in Turkey. In addition, we compared the clinicopathological data of mCRC patients with the mutational status of KRAS and BRAF in cancerous tissues from these patients.

\section{Materials and Methods}

\section{Patients}

This retrospective study enrolled 50 patients with a histopathologically proven metastatic colorectal cancer diagnosis at the Uludağ University Faculty of Medicine, Department of Medical Genetics. The patients were evaluated for mutations of KRAS codon 12, 13 and 61 and BRAF codon 600. The demographic (age, gender) and histopathological features (tumor localization, growth pattern, histological type, differentiation and clinical grading) were compared with the results of molecular analyses. The local ethics committee approved this study.

\section{Genotyping}

The DNA used for sequence analyses were isolated from paraffin embedded colorectal tumor tissues using the QIAmp DNA FFPE Tissue Kit. For KRAS codon 12 and 13 regions the primers were the following: $F$ : 5'-GGTACTGGTGGAGTATTTGATAGTG-3' and R: 5'-TGAAACCCAAGGTACATTTCAG-3' for codon 61 F: 5'-TTTGTATTAAAAGGTACTGGTGGAG-3' and R: 5'-CCTTTATCTGTATCAAAGAATGGTC-3' primers. For the BRAF codon 600 region the primers were F: 5'-TCTTACCTAAACTCTTCATAATGCTTG-3 and R: 5'-GACTTTCTAGTAACTCAGCAGCATC-3'. The sequences were designed with the primer3 software program (http://ihg.gsf.de/ihg/ExonPrimer.html). The related regions were amplified by PCR using H-taq polymerase enzyme and the primers. Standard PCR conditions with 35 cycles were used and the reactions were performed on a 9700 Thermal Cycler (Applied Biosystems). The amplified products were purified by using Zymo Research Sequencing Clean-up Kit (The Epigenetic Company, Irvine, USA). The cycle sequencing was performed with Bigdye Terminator v3.1 Cycle Sequencing Kit (Applied Biosystems, Foster City, USA) on a 3130 Genetic Analyzer (Applied Biosystems, Foster City, USA). The sequence data were analyzed using the sequencing analysis v5.3.1 software program (Applied Biosystems, Foster City, USA) and compared to the reference sequence (GenBank Accession Nos. KRAS; NM_004985.3, NG_007524.1 BRAF; NM_004333.4, NG_007873.2).

\section{Statistical Analyses}

The age variable was defined by median (minimummaximum) values and the categorical variables are shown as numbers and related percentages. The Pearson chisquare and Fisher's exact chi-square tests were used for the comparisons of categorical variables between mutation groups. The analyses were performed using the SPSS 13.0 (Chicago, IL.) program and $\mathrm{p}<0.05$ was accepted as statistically significant.

\section{Results}

Of the $50 \mathrm{mCRC}$ patients enrolled in this study, 29 was male $(58 \%), 21$ were female $(42 \%)$. The male/female ratio was 1.38 . The median age of the patients was $58(30-76)$ years. The mean age of the male patients was $57 \pm 10.98$, and the mean age of the female patients was $54.67 \pm 11.45$. The clinicopathological features of the patients with mCRC are shown in Table 1.

KRAS and BRAF mutations were identified in 15 $(30 \%)$ and $1(2 \%)$ patients, respectively. We found KRAS mutations in codon 12,13 , and 61 , in $73.33 \%(11 / 15)$, $20 \%(3 / 15)$ and $6.67 \%(1 / 15)$ of patients, respectively. The codon 12 mutations were found to be the following: $33.33 \%$ Gly 12 Asp (5/15), 26.66\% Gly 12Val (4/15), 6.67\% Gly12Cys (1/15), 6.67\% Gly12Ala (1/15). The codon 13 mutations were Gly13Asp (3/15) in $20 \%$ of patients (Figure 1). The codon 61 mutation was Gln61His (1/15) and occurred in $6.67 \%$ of patients (Figure 2) We detected a heterozygous shift from GTG to GAG in codon 600 of

\section{Table 1. Histopathological Features of the Patients}

\begin{tabular}{lc}
\hline Histopathologic Features & Number $(\%)$ \\
Tumor Localization & \\
\hline Ceacum & $4(8)$ \\
Ascending colon & $8(16)$ \\
Transverse colon & $2(4)$ \\
Descending colon & $4(8)$ \\
Sigmoid colon & $7(14)$ \\
Rectosigmoid colon & $5(10)$ \\
Rectum & $20(40)$ \\
Histological Type & \\
Adenocarcinoma & $46(92)$ \\
Mucinous adenocarcinoma & $4(8)$ \\
Differentiation & \\
Moderate differentiation & $43(86)$ \\
Poor differentiation & $7(14)$ \\
Growth Pattern & \\
Polypoid & $5(10)$ \\
Ulceroinfiltrative & $10(20)$ \\
Ulserovegetative & $30(60)$ \\
Annuler & $1(2)$ \\
Infiltrative & $3(6)$ \\
Ulserative & $1(2)$ \\
\hline
\end{tabular}
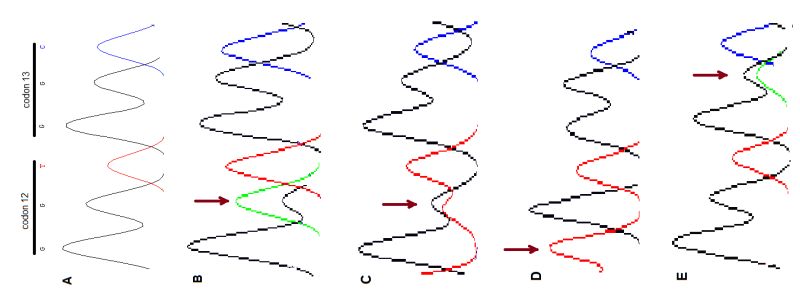

Figure 1. Electropherogram for KRAS codon 12 and codon 13 mutants. A: Wild type codon 12 and codon 13, B: p.G12D, codon $12 \mathrm{GGT}>\mathrm{GAT}, \mathrm{c} .35 \mathrm{G}>\mathrm{A}, \mathrm{C}$ : p.G12V, codon 12 GGT $>$ GTT, c. $35 \mathrm{G}>\mathrm{T}$, D: p.G12C, codon 12 GGT $>$ TGT, c.34 G>T, E: p.G13D, codon 13 GGC $>$ GAC, c.38G $>$ A 
Table 2. Correlation of KRAS Mutation Status with Clinical and Histopathological Features

\begin{tabular}{|c|c|c|c|}
\hline & $\begin{array}{l}\text { Wild Type number }(\%) \\
\qquad \mathrm{n}=35\end{array}$ & $\begin{array}{l}\text { Mutation Carrier number (\%) } \\
\qquad \mathrm{n}=15\end{array}$ & p-value \\
\hline Age & $59(32-76)$ & $57(30-70)$ & 0.539 \\
\hline Gender (Male/Female) & $21(60) / 14(40)$ & $8(53.3) / 7(46.7)$ & 0.900 \\
\hline \multicolumn{4}{|l|}{ Tumor Location } \\
\hline Caecum & $2(5.7)$ & $2(13.3)$ & 0.574 \\
\hline Ascending colon & $3(8.6)$ & $5(33.3)$ & 0.043 \\
\hline Transverse colon & $2(5.7)$ & $0(0)$ & 1,000 \\
\hline Descending colon & $4(11.4)$ & $0(0)$ & 0.302 \\
\hline Sigmoid colon & $6(17.1)$ & $1(6.7)$ & 0.659 \\
\hline Rectosigmoid colon & $4(11.4)$ & $1(6.7)$ & 1,000 \\
\hline Rectum & $14(40)$ & $6(40)$ & 0.753 \\
\hline \multicolumn{4}{|l|}{ Growth Pattern } \\
\hline Polypoid & $3(8.6)$ & $2(13.3)$ & 0.629 \\
\hline Ulceroinfiltrative & $7(20)$ & $3(20)$ & 1,000 \\
\hline Ulcerovegetative & $21(60)$ & $9(60)$ & 0.752 \\
\hline Annuler & $1(2.9)$ & $0(0)$ & 1,000 \\
\hline Infiltrative & $2(5.7)$ & $1(6.7)$ & 1,000 \\
\hline Ulserative & $1(2.9)$ & $0(0)$ & 1,000 \\
\hline \multicolumn{4}{|l|}{ Histological Type } \\
\hline Adenocarsinom & $32(91.4)$ & $14(93.3)$ & \multirow{2}{*}{1} \\
\hline Musinous adenocarcinoma & $3(8.6)$ & $1(6.7)$ & \\
\hline \multicolumn{4}{|l|}{ Grade } \\
\hline Moderate differentiation & $29(82.9)$ & $14(93.3)$ & \multirow{2}{*}{0.659} \\
\hline Poor differentiation & $6(17.1)$ & $1(6.7)$ & \\
\hline
\end{tabular}

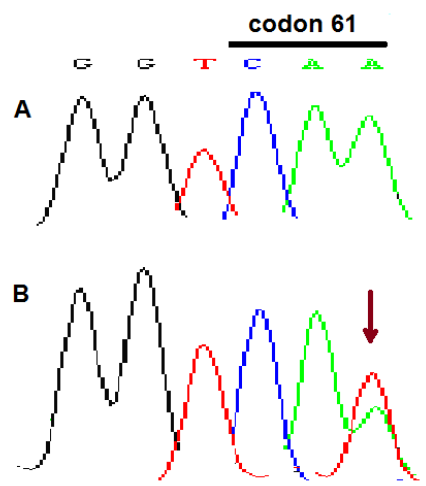

Figure 2. Electropherogram for KRAS Codon 61 Mutation. A: Wild type codon 61, B: p.Q61H, codon 61 CAA $>$ CAT, c. $183 \mathrm{~A}>\mathrm{T}$

the BRAF gene (p.V600E) in one patient (Figure 3).

We compared the clinicopathological features of the patients with KRAS mutations and wild types and found the median age was 59 (32-76) for wild types and 57 (30-70) for patients with mutations. There was no significant difference between the groups in terms of age. We classified the tumor locations into the following sites: cecum, ascending colon, transverse colon, sigmoid, rectosigmoid junction and the rectum. The presence of KRAS mutation was significantly higher in patients with tumors in the ascending colon $(\mathrm{p}=0.043)$. The tumors on the right side of the colon (cecum, ascending and transverse colon) are grouped together and compared with those on the left (splenic flexure to rectum). This analysis showed that $50 \%$ of the right colon tumors had KRAS mutations, whereas only $22 \%$ of the left side tumors had

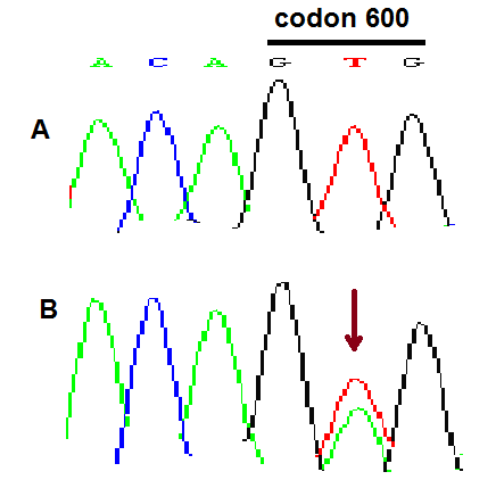

Figure 3. Electropherogram for BRAFcodon 600 Mutation. A: Wild type codon 600, B: p.V600E, codon 600 GTG $>$ GAG, c.1799T $>$ A

the same mutation $(\mathrm{P}=0.085)$. The frequencies of KRAS codon 12 mutations were $54.5 \%$ and $45.5 \%$ in the right and left colon, respectively. The frequencies of KRAS codon 13 mutations were $33.3 \%$ and $66.7 \%$ in the right and left colon, respectively. There was no statistically significant relationship between codon 12 and 13 mutations and the tumor locations. We did not find other significant associations between KRAS mutation and the patients' clinicopathological characteristics (Table 2).

\section{Discussion}

Although the discovery of KRAS mutations affects individual treatment regimens in metastatic CRC patients, the KRAS mutation type and aberrations of related proteins, such as BRAF, are also important for patient 
selection and study outcomes (Patel and Karapetis, 2013). Cetuximub and panitumumab are the major therapies used against EGFR in patients with CRC. These drugs bind to EGFR and inhibit tumor growth, invasion and angiogenesis. However, gene mutations involving BRAF and KRAS in the EGFR pathway abolish the response to anti-EGFR treatment in patients with CRC. Thus, these gene aberrations are predictive markers for failure of EGFR targeted therapies (Chang et al., 2013).

In previous studies, KRAS mutations were found in $40 \%$ of the patients with CRC, and the most frequent mutation type was the missense point mutation. The point mutation regions of the KRAS gene are mostly found in codon 12 and $13(\sim 80 \%$ and $\sim 17 \%$, respectively), and the other regions are less affected (Brand and Wheeler, 2012; Marchoudi et al., 2013; Patel and Karapetis, 2013; Ferreira et al., 2014). Zlobec et al., (2010) found KRAS gene (codon 12 or 13 ) mutations in $30.1 \%$ of 404 CRC patients. Ferreira et al. (2014) found KRAS codon 12 and 13 mutations in $31.9 \%$ of $8234 \mathrm{mCRC}$ patients. They detected the Gly12Asp (GGT>GAT) mutation on codon 12 most frequently and Gly 13Asp (GGC>GAC) mutation on codon 13. Chang et al. (2013) found mutations in KRAS codon 12 and 13 in $35.76 \%$ of 165 CRC patients and did not find mutations in codon 61 . They found that the majority of the mutations occurred in codon 12 and the most frequent mutation in codon 12 was reported to be GGT>GAT. Shen et al. (2013) found KRAS mutations in $35.9 \%$ of $676 \mathrm{CRC}$ patients and these mutations were in codon 12,13 and 61 at frequencies of $25.7 \%, 6.8 \%$ and $2.1 \%$, respectively. The most frequent mutations in codon 12 and 13 were G12D and G13D, respectively. Tong et al., (2014) found KRAS mutations in $44.5 \%$ of 1506 patients and these mutations were in codon 12,13 and 61 at frequencies of $75.1 \%, 19.3 \%$ and $2.5 \%$, respectively. The most frequent mutation in codon 12 was G12D and the most frequent mutation in codon 13 was G13D. Ozen et al. (2013) found KRAS mutations in $49.05 \%$ of 53 CRC patients, and these were in codon 12,13 and 61 with frequencies of $65.38 \%, 26.93 \%$ and $7.69 \%$, respectively. We detected KRAS mutations in $30 \%$ of the patients with mCRC, and these were in codon 12, 13 and 61 at frequencies of $73.33 \%, 20 \%$ and $6.67 \%$, respectively. We found the Gly12Asp (GGT>GAT) mutation in codon 12 to be the most frequent mutation by $33.33 \%$ and the Gly13Asp (GGC>GAC) mutation is the only mutation found in patients who have codon 13 mutations. We detected a KRAS mutation in codon 61 in one patient (Gln61His, CAA >CAT). Our results are compatible with data reported in the literature.

Tong et al. (2014) found KRAS mutations to be significantly higher in the female and right colon tumors. Furthermore, they detected more frequent mutations in codon 12 in the left colon and codon 13 mutations were more frequent in the right colon. The location of KRAS mutations may be related to different molecular pathways in patients with CRC in the left or right colon. The right and left colon tumors are distinct tumoral structures due to their epidemiological, clinicopathological and molecular biological features. Right colon cancer is associated with female gender, advanced age, advanced stage and poorly differentiated mucinous histological type (Lin et al., 2006; Benedix et al., 2010; Pai et al., 2012; Rosty et al., 2013). However, no relationship was established with mucinous histology in our study. The presence of higher microsatellite instability and KRAS mutations are frequent molecular characteristics of right colon cancers. Chromosomal instability and TP53 mutations are more frequent in left-sided tumors (Bufill, 1990; Soong et al., 2000; Iacopetta, 2002; Sugai et al., 2006). The cause of the differences between left and right-sided colon adenocarcinomas remains ambiguous. The differences may be multifactorial and could involve embryological origin and the effects of chemical and bacterial luminal microenvironments (Tong et al., 2014). Yokota et al. (2011) found KRAS gene (codon 12 and 13) mutations in $34.5 \%$ of 229 advanced stage and relapsed CRC patients and showed the frequency of right colon localization was significantly higher in patients with tumors harboring KRAS codon 13 mutations. Samowitz et al. (2000) detected KRAS mutation in $31.8 \%$ of 1413 patients with colon cancer and found that these mutations were more frequent in the proximal (right) colon compared to the distal (left) colon. We also found KRAS mutations are significantly more frequent in the ascending colon and the mutation frequency was higher in tumors of the right colon compared to the left.

BRAF gene mutations could be used as a biomarker for anti-EGFR monoclonal antibody resistance. The BRAF gene mutation ratios vary from 1.7 to $13 \%$. Chang et al., (2013) detected BRAF gene (V600E) mutations in $4.24 \%$ of patients. Shen et al. (2013) found BRAF mutation in $6.96 \%$ of patients and V600E mutations $1.8 \%$ of patients. Ozen et al. (2013) found no BRAF mutation in their patients. However, Yokota et al. (2011) found mutations of the BRAF gene (codon 600) in 6.5\% of patients. They found that the BRAF mutation carriers had right colon tumors significantly more frequently and stated that BRAF gene mutation status was a strong prognostic factor in advanced stage and relapsed CRC patients. Zlobec et al. (2010) detected V600E mutation in a $12 \%$ of cases and indicated that this mutation was a poor prognostic factor for CRC patients with tumors in the right colon. These differences between studies may stem from ethnic heterogeneity, study design differences and the characteristics of the patients enrolled in the studies. We detected BRAF gene V600E mutation in $2 \%(n=1)$ of cases in our study. However, we could not associate the V600E mutation with clinicopathological parameters because of the low number of cases with this mutation.

In this study, we found KRAS and BRAF mutations in $30 \%$ and $2 \%$ of patients, respectively. We found that KRAS mutations are related to tumors of the ascending colon. Future studies of larger patient groups would provide more accurate information regarding the relationship between KRAS and BRAF mutations and the clinicopathological features in patients with CRC.

\section{References}

Benedix F, Kube R, Meyer F, et al (2010). Comparison of 17,641 patients with right- and left-sided colon cancer: differences in 
epidemiology, perioperative course, histology, and survival. Diseases Colon Rectum, 53, 57-64.

Brand T, Wheeler D (2012). KRAS mutant colorectal tumors: past and present. Small GTPases, 3, 34-9.

Brenner H, Kloor M, Pox C (2014). Colorectal cancer. Lancet, 383, 1490-502.

Bufill J (1990). Colorectal cancer: evidence for distinct genetic categories based on proximal or distal tumor location. Ann Internal Med, 113, 779-88.

Chang Y, Chang S, Yeh K, et al (2013). RAS, BRAF, and TP53 gene mutations in Taiwanese colorectal cancer patients. Onkologie, 36, 719-24.

Ciardiello F, Tortora G (2008) Drug therapy: EGFR antagonists in cancer treatment. New Engl J Med, 358, 1160-74.

Cunningham D, Humblet Y, Siena S, et al (2004). Cetuximab monotherapy and cetuximab plus irinotecan in irinotecanrefractory metastatic colorectal cancer. New Engl J Med, 351, 337-45.

Di Nicolantonio F, Martini M, Molinari F, et al (2008). Wild-type $\mathrm{BRAF}$ is required for response to panitumumab or cetuximab in metastatic colorectal cancer. J Clin Oncol, 26, 5705-12.

Ferreira C, Aran V, Zalcberg-Renault I, et al (2014). KRAS mutations: variable incidences in a Brazilian cohort of 8,234 metastatic colorectal cancer patients. Bmc Gastroenterol, 14.

Friday B, Adjei A (2008). Advances in targeting the Ras/Raf/ MEK/Erk mitogen-activated protein kinase cascade with MEK inhibitors for cancer therapy. Clin Cancer Res, 14, 342-46.

Iacopetta B (2002). Are there two sides to colorectal cancer? Int J Cancer, 101, 403-8.

Jean G, Shah S (2008). Epidermal growth factor receptor monoclonal antibodies for the treatment of metastatic colorectal cancer. Pharmacotherapy, 28, 742-54.

Lin J, Chang S, Wang H, et al (2006). Distinctive clinicopathological features of Ki-ras mutated colorectal cancers. J Surgical Oncol, 94, 234-41.

Marchoudi N, Joutei H, Jouali F, et al (2013). Distribution of KRAS and BRAF mutations in Moroccan patients with advanced colorectal cancer. Pathologie Biologie, 61, 273-6.

Ozen F, Ozdemir S, Zemheri E, et al (2013). The proto-oncogene kras and braf profiles and some clinical characteristics in colorectal cancer in the turkish population. Genetic Testing Molecular Biomarkers, 17, 135-9.

Pai R, Jayachandran P, Koong A, et al (2012). BRAF-mutated, microsatellite-stable adenocarcinoma of the proximal colon: an aggressive adenocarcinoma with poor survival, mucinous differentiation, and adverse morphologic features. American J Surgical Pathol, 36, 744-52.

Patel G, Karapetis C (2013). Personalized treatment for advanced colorectal cancer: KRAS and beyond. Cancer Manage Res, 21, 387-400.

Roberts P, Der C (2007). Targeting the Raf-MEK-ERK mitogenactivated protein kinase cascade for the treatment of cancer. Oncogene, 26, 3291-310.

Rosty C, Young J, Walsh M, et al (2013). Colorectal carcinomas with KRAS mutation are associated with distinctive morphological and molecular features. Modern Pathol, 26, 825-34.

Samowitz W, Curtin K, Schaffer D, et al (2000). Relationship of Ki-ras mutations in colon cancers to tumor location, stage, and survival: A population-based study. Cancer Epidemiol Biomarkers Prev, 9, 1193-7.

Shen Y, Wang J, Han X, et al (2013). Effectors of epidermal growth factor receptor pathway: the genetic profiling of KRAS, BRAF, PIK3CA, NRAS mutations in colorectal cancer characteristics and personalized medicine. Plos One, 8.
DOI:http://dx.doi.org/10.7314/APJCP.2016.17.3.1175 KRAS and BRAF Mutations in Metatstatic Colorectal Cancer

Soong R, Powell B, Elsaleh H, et al (2000). Prognostic significance of TP53 gene mutation in 995 cases of colorectal carcinoma: influence of tumour site, stage, adjuvant chemotherapy and type of mutation. Eur J Cancer, 36, 2053-60.

Sugai T, Habano W, Jiao Y, et al (2006). Analysis of molecular alterations in left- and right-sided colorectal carcinomas reveals distinct pathways of carcinogenesis - Proposal for new molecular profile of colorectal carcinomas.J Molecular Diagnostics, 8, 193-201.

Tong J, Lung R, Sin F, et al (2014). Characterization of rare transforming KRAS mutations in sporadic colorectal cancer. Cancer Biology Therapy, 15, 768-76.

Venook A (2005), Epidermal growth factor receptor-targeted treatment for advanced colorectal carcinoma. Cancer, 103, 2435-46.

Yokota T (2012). Are KRAS/BRAF mutations potent prognostic and/or predictive biomarkers in colorectal cancers? AntiCancer Agents in Medicinal Chemistry, 12, 163-71.

Yokota T, Ura T, Shibata N, et al (2011). BRAF mutation is a powerful prognostic factor in advanced and recurrent colorectal cancer. British J Cancer, 104, 856-62.

Zlobec I, Bihl M, Schwarb H, et al (2010). Clinicopathological and protein characterization of BRAF- and K-RAS-mutated colorectal cancer and implications for prognosis. Int $J$ Cancer, 127, 367-80. 